\title{
Dinámica del plancton en la primera milla náutica frente a la provincia de El Oro, Ecuador
}

\author{
Jessica Salcedo ${ }^{1,2} \&$ Dialhy Coello ${ }^{1,3}$
}

1. Investigación de Recursos Bioacuáticos y su Ambiente, Instituto Nacional de Pesca, Letamendi 102 y la Ría, Guayaquil, Ecuador.

2. Escuela Superior Politécnica del Litoral, ESPOL, Centro Nacional de Acuicultura e Investigaciones Marinas, CENAIM, Campus Gustavo Galindo Km 30.5 Via Perimetral, P.O. Box 09-01-5863, Guayaquil, Ecuador; jsalcedo@espol.edu.ec

3. Docente de la Facultad de Ciencias Naturales, Universidad de Guayaquil. Av. Raúl Gómez Lince s/n y Av. Juan Tanca Marengo. Guayaquil, Ecuador; dialhy.coellos@ug.edu.ec

Recibido 14-VIII-2017. Corregido 22-II-2018. ～Aceptado 20-III-2018.

\begin{abstract}
Plankton dynamics in the first nautical mile of El Oro province, Ecuador. El Oro includes different coastal and estuarine systems and the important Gulf of Guayaquil. These areas provide different ecosystem services, but with the increase of population and of the varied economic activities, the barely known local natural resources may be negatively impacted. Thus this research aimed to study plankton dynamics, productivity and ecological balance for the area during the dry season of 2012. For this, we established 17 stations and studied the monthly variation and spatial distribution of inorganic nutrients, temperature, dissolved oxygen, salinity, photic zone, plankton and fish larvae abundance and composition with standard methods, while spatial and temporal patterns where identified using cluster and multidimentional scaling analyses. Obtained results of biotic and abiotic parameters defined a pattern characteristic of productive estuarine zones as well as the existence of three specific areas associated with the natural and anthropic conditions of the area, such as: a) inner part of the Jambelí Archipelago and in front of Puná island influenced by productive activities and the hydrography of the area; B) an area, formed by the stations located in the outer estuary where the oceanic influence is evident and c) the Jambelí channel, characterized by mixing processes resulting from tides flow. This was supported by the presence of oceanic, neritic and estuarine phytoplankton; copepods with uniform distribution in the study area, and dominance of Engraulidae fish larvae, representative of this type of aquatic ecosystem. Temporally, rainfall records in May exceeded normal values, which would have influenced the high concentrations of nutrients, decrease of salinity and of the photic zone registered during this month. However, the availability of nutrients throughout the study period, allowed to maintain the composition and abundance of phytoplankton, with changes in representative species, recorded periods of greater (June to August) and lower (October to December) zooplankton abundance, could be the result of specific population patterns of present species, and the influence of the tide and the flows of the contributing river basin. A similar behavior was recorded for ichthioplankton, as a result of Anchoa macrolepidota concentrations, species, characteristics of this type of ecosystem. These patterns of spatio-temporal distribution, allowed the development of multiple abiotic and biotic interactions that determine different strategies to maintain the productivity of the area. We recommend new studies during the rainy season in Ecuador. Rev. Biol. Trop. 66(2): 836-847. Epub 2018 June 01.
\end{abstract}

Key words: plankton; Gulf of Guayaquil; dry season; biotic and abiotic variables.

Los diferentes grupos que componen el plancton son elementos funcionales básicos en los ecosistemas, y sus variaciones en el tiempo y espacio, están influenciadas por interacciones intraespecíficas e interespecíficas, especialmente en zonas de surgencia costera, donde la circulación asociada tiene un efecto regional e incluso local sobre el fitoplancton (biomasa y tasas fotosintéticas) y por ende en el zooplancton (Palma, Henríquez, \& Ojeda, 2009).

La primera milla náutica a lo largo del perfil costero ecuatoriano incluye sistemas 
costeros y estuarinos que determinan la formación de diferentes hábitats como playas, bosques de manglar, bajos arenosos-lodosos, costas rocosas, acantilados y humedales costeros y la presencia de procesos locales de surgencia (Hurtado, Hurtado-Domínguez, Hurtado-Domínguez, Soto, \& Merizalde, 2010; Vera, Lucero, \& Mindiola, 2009). Esta brinda múltiples servicios ecosistémicos, especialmente, explotación de recursos naturales con fines productivos y de subsistencia, que en algunos casos están ligados a afectaciones negativas, por formas inadecuadas de aprovechamiento, entre ellas la sobrepesca y la deforestación (Coello \& Altamirano, 2007).

A pesar de la importancia de esta zona dentro del ecosistema marino costero, existe limitada información referente a los aspectos que determinan la productividad y el equilibrio ecológico de la misma. Domínguez y Fockedey (2001), determinaron la presencia de peces de interés comercial como mojarras (Gerridae: Gerres cinereus, Eucinostomus spp.), anchoas (Engraulidae: Engraulis ringens, Anchoa sp.), pejereyes (Atherinidae: Atherinidae sp., Artherinopsis californiensis, Nectarges sp.), así como el uso de esta área como región de desarrollo y alimentación de la gran mayoría de migrantes mareales, entre ellos larvas de camarón (Penaeidae), cangrejos de mar (braquiurus) y peces.

Uno de los accidentes geográficos más importantes del perfil costero ecuatoriano y de la costa del Pacífico Sur, es el Golfo de Guayaquil, el cual con base en la información generada entre las décadas del 70 al 90, presenta variaciones espaciales y temporales en la concentración de fosfato, silicato y nitrato, además de alta diversidad biológica asociada al desarrollo del Frente Ecuatorial, afloramientos costeros e interacción de diversos tipos de masas de aguas oceánicas y continentales (Aguirre \& Shervette, 2005; Cucalón, 1996; Jiménez, 1996).

Por la importancia productiva y ecosistémica de esta zona, se desarrolló esta investigación, con el objetivo de describir la dinámica del plancton durante la estación climática seca del 2012, en la parte sur del golfo de Guayaquil. Para esto se analizó la variación mensual y espacial de los nutrientes inorgánicos, oxígeno disuelto, temperatura, salinidad, zona fótica y la abundancia y composición del fitoplancton, zooplancton y larvas de peces.

\section{MATERIALES Y MÉTODOS}

El área de estudio comprendió principalmente estaciones dentro de la primera milla náutica frente a la provincia de El Oro, específicamente al sur del golfo de Guayaquil $\left(02^{\circ} 57.356\right.$ - $03^{\circ} 25.122 \mathrm{~S} \& 079^{\circ} 54.161$ $\left.080^{\circ} 22.674 \mathrm{~W}\right)$. Éste es un complejo y dinámico sistema de transición en el que convergen los aportes del mayor sistema hidrográfico de la costa ecuatoriana (río Guayas) y las principales masas de agua del Pacífico Oriental (Hurtado et al., 2010). El periodo analizado fue junio-diciembre 2012, que incluye a la estación considerada como seca en el Ecuador, aunque según algunos autores, junio y diciembre pueden ser considerados de transición entre periodos estacionales (Moreano, 1983; Cucalón, 1996).

Para desarrollar la investigación se estableció un monitoreo mensual que comprendió 17 estaciones (Cuadro 1), en las cuales se obtuvieron muestras y datos de agua a nivel superficial $(0.50 \mathrm{~m})$ para la determinación de variables físico-químicas y planctónicas.

La temperatura y salinidad fueron determinadas mediante perfiladores (CTD) (Seabird 19 y 19 plus). La profundidad de la zona eufótica (Zeu), fue calculada en base a la profundidad del disco Secchi y la fórmula descrita por Kirk (1994). Las muestras de agua para el análisis de fitoplancton y determinación de variables químicas fueron obtenidas mediante botellas muestreadoras Niskin de cinco litros de capacidad. Para la determinación de oxígeno disuelto la muestra se almacenó en botellas DBO de vidrio transparente de $300 \mathrm{ml}$ de capacidad y se analizó por el método de Winkler modificado por Carpenter (1965). Otro volumen de la muestra fue envasada en frascos color ámbar de polietileno de $1000 \mathrm{ml}$ y filtrados al vacío 
CUADRO 1

Posición geográfica de las estaciones en la primera milla náutica de la Provincia de El Oro, Ecuador (junio-diciembre 2012)

TABLE 1

Geographical position of the sampling stations in the first mile in front of El Oro province, Ecuador (June-December 2012)

\begin{tabular}{cccccc} 
Estación & Latitud & Longitud & Estación & Latitud & Longitud \\
\hline 1 & $03^{\circ} 24.176 \mathrm{~S}$ & $080^{\circ} 17.917 \mathrm{~W}$ & 10 & $03^{\circ} 12.109 \mathrm{~S}$ & $080^{\circ} 02.344 \mathrm{~W}$ \\
2 & $03^{\circ} 25.122 \mathrm{~S}$ & $080^{\circ} 09.731 \mathrm{~W}$ & 11 & $03^{\circ} 10.662 \mathrm{~S}$ & $080^{\circ} 07.281 \mathrm{~W}$ \\
3 & $03^{\circ} 23.858 \mathrm{~S}$ & $080^{\circ} 03.793 \mathrm{~W}$ & 12 & $03^{\circ} 03.674 \mathrm{~S}$ & $080^{\circ} 11.000 \mathrm{~W}$ \\
4 & $03^{\circ} 17.090 \mathrm{~S}$ & $080^{\circ} 01.510 \mathrm{~W}$ & 13 & $03^{\circ} 08.664 \mathrm{~S}$ & $080^{\circ} 02.415 \mathrm{~W}$ \\
5 & $03^{\circ} 10.385 \mathrm{~S}$ & $080^{\circ} 22.674 \mathrm{~W}$ & 14 & $03^{\circ} 09.256 \mathrm{~S}$ & $079^{\circ} 57.323 \mathrm{~W}$ \\
6 & $03^{\circ} 14.759 \mathrm{~S}$ & $080^{\circ} 15.184 \mathrm{~W}$ & 15 & $02^{\circ} 57.356 \mathrm{~S}$ & $080^{\circ} 03.989 \mathrm{~W}$ \\
7 & $03^{\circ} 19.862 \mathrm{~S}$ & $080^{\circ} 14.110 \mathrm{~W}$ & 16 & $03^{\circ} 00.274 \mathrm{~S}$ & $079^{\circ} 59.318 \mathrm{~W}$ \\
8 & $03^{\circ} 14.552 \mathrm{~S}$ & $080^{\circ} 07.119 \mathrm{~W}$ & 17 & $03^{\circ} 01.276 \mathrm{~S}$ & $079^{\circ} 54.161 \mathrm{~W}$ \\
9 & $03^{\circ} 16.195 \mathrm{~S}$ & $080^{\circ} 04.330 \mathrm{~W}$ & & & \\
\hline
\end{tabular}

mediante filtros de fibra de vidrio Wathman $\mathrm{GF} / \mathrm{C}$, el líquido sobrenadante se almacenó y se llevó a congelación $\left(4^{\circ} \mathrm{C}\right)$, para posteriormente determinar en el laboratorio nitrito, nitrato, fosfato y silicato disueltos según las metodologías descritas en Standard Methods (Rice, Baird, Eaton, \& Clesceri, 2012).

Las muestras de fitoplancton $(230 \mathrm{ml})$ fueron preservadas in situ con una solución de Lugol al $0.5 \%$ y cuantificadas a partir de una submuestra mediante el método de Utermöhl (Reguera, Alonso, Moreira, \& Méndez, 2011), obteniéndose los resultados en cel.L $\mathrm{L}^{-1}$. Para el zooplancton y larvas de peces se realizaron arrastres superficiales a dos nudos de velocidad de cinco minutos de duración con redes planctónicas WP2 (60 cm de diámetro y 200 y $300 \mu \mathrm{m}$ de apertura de malla); las muestras fueron preservadas en una solución de formol al $4.0 \%$ (Boltovskoy, 1981). Su cuantificación se realizó con cámaras de Dolfus en el caso de zooplancton con resultados expresados en org. $\mathrm{m}^{-3}$, y la metodología de Smith and Richardson (1979) para el ictioplancton; este valor fue estandarizado al número de larvas por arrastre, respecto una unidad de medida, es decir, larvas. $10 \mathrm{~m}^{-2}$ de superficie de mar.

Para la identificación taxonómica del fitoplancton, se realizó hasta el menor nivel taxonómico posible, con base en los trabajos de Al-Kandari, Al-Yamani y Al-Rifaie (2009);
Rodríguez, Hernández y Lizárraga (2008); Siqueiros-Beltrones (2002); Soler, Pérez y Aguilar (2003); Tomas, (1996); Moreno, Licea y Santoyo (1996); Licea, Moreno, Santoyo y Figueroa (1995). Para el zooplancton, se empleó Suárez-Morales (1995, 1996); Björnberg, (1981) y Boltovskoy (1981); mientras que para el ictioplancton, se consideró los caracteres merísticos descritos en las claves de Calderón (2011), Beltrán-León y Ríos (2000) y Ahlstrom (1972). Todas las muestras analizadas se encuentran en el Instituto Nacional de Pesca del Ecuador.

Los análisis estadísticos se realizaron utilizando los programas R (R Core TEAM 2014) y PRIMER v6 package (Clarke \& Gorley, 2005). Previo al análisis de los datos, los valores de las variables físicas (salinidad, temperatura, oxígeno disuelto y profundidad de la zona eufótica) y químicas (nitrito, nitrato, fosfato, silicato) fueron transformados mediante una función logarítmica y estandarizados para poner todas las variables dentro de la misma escala. Los datos planctónicos fueron transformados con una función de raíz cuadrada, para disminuir el peso de las especies cuantitativamente dominantes en los análisis (Clarke \& Gorley, 2005).

Para identificar patrones espaciales y temporales en la distribución y composición de especies, así como en los parámetros abióticos, se aplicó un análisis de conglomerados 
(CLUSTER) basado en el método del promedio de grupo. La matriz de semejanzas se calculó en base al coeficiente de semejanza de BrayCurtis para los datos bióticos y la distancia euclidiana para los datos abióticos. Para determinar diferencias estadísticas en los conglomerados, se aplicó un test de permutación de perfiles de semejanza (SIMPROF, $\mathrm{P}<0.05$ ), los resultados obtenidos se representaron mediante análisis de ordenamiento multidimensional (MDS) (Clarke \& Gorley, 2005).

Para determinar si existieron variaciones espaciales y temporales significativas en las variables estudiadas, se aplicó la prueba estadística de Anova de una vía, con comparación múltiple de Tukey, y en el caso de no cumplir con los requisitos de normalidad (Shapiro-wilcoxon, $\alpha=0.05)$ y de homogeneidad de varianzas (Levene, $\alpha=0.05$ ), se aplicó la prueba no paramétrica de Kruskal Wallis (Zar, 2009).

Para los análisis realizados, se utilizó en el caso del fitoplancton y larvas de peces, información a nivel de especie, mientras que para el zooplancton, debido al bajo porcentaje de organismos identificados a especie (35.2\%), se empleó la información correspondiente a taxones gruesos (órdenes).

\section{RESULTADOS}

Variación espacial: Los valores promedio de nitrito fluctuaron entre $0.003 \pm 0.003$ (estaciones 5 y 6 ) y $0.182 \pm 0.048 \mathrm{mg} . \mathrm{L}^{-1}$ (estación 14); el nitrato osciló entre $0.051 \pm 0.039$ (estación 5) y $0.860 \pm 0.042 \mathrm{mg} . \mathrm{L}^{-1}$ (estación 17), y el silicato tuvo su menor valor en la estación 5 con $0.451 \pm 0.535 \mathrm{mg} . \mathrm{L}^{-1}$ y el máximo en la estación 3 con $4.161 \pm 3.300 \mathrm{mg} . \mathrm{L}^{-1}$. La mínima concentración de fosfato correspondió a la estación 5 con $0.050 \pm 0.034 \mathrm{mg} . \mathrm{L}^{-1}$, pero su máximo fue determinado en la estación 2 con $0.172 \pm 0.031 \mathrm{mg} . \mathrm{L}^{-1}$. En términos generales, la estación siete localizada al suroeste de la isla Puná, registró la menor concentración de nutrientes, mientras que las mayores concentraciones de nutrientes fueron registradas en estaciones cercanas al margen continental (3, 14 y 17) (APÉNDICE 1A).
En relación con el oxígeno disuelto los valores promedio fluctuaron entre $4.429 \pm$ 1.186 y $8.764 \pm 0.776 \mathrm{mg} . \mathrm{L}^{-1}$ en las estaciones 3 y 6 , correspondientes al interior del estuario de Jambelí y al estuario exterior del Golfo de Guayaquil, respectivamente. La temperatura registró un promedio mínimo de $24.29 \pm 0.89$ ${ }^{\circ} \mathrm{C}$ en la estación 12 y un máximo de 26.49 $\pm 1.16{ }^{\circ} \mathrm{C}$ en la estación 17 ; mientras que la salinidad fluctuó entre $30.50 \pm 3.05$, y $33.78 \pm$ 0.83 ups en las estaciones 17 y cinco, respectivamente. Finalmente, la mayor profundidad promedio de la zona eufótica fue de $17.36 \pm$ $10.04 \mathrm{~m}$ en la estación $5 \mathrm{y}$ la mínima de 1.57 $\pm 0.45 \mathrm{~m}$ en la estación 12 (APÉNDICE 1B).

El perfil de similitud identificó cinco grupos en el área de estudio, el primero conformado por las estaciones 12, 15 y 16, ubicadas en la parte sur y este de la isla Puna; el segundo grupo, lo conformaron las estaciones 8 a 11, 13, 1 y 7, las dos últimas ubicadas al sur del estuario de Jambelí y las otras en el canal del mismo nombre, lo que sugiere una distribución uniforme de los parámetros abióticos desde la parte oceánica hacia el interior del estuario del Golfo de Guayaquil (Fig. 1A).

El tercer grupo lo conformaron las estaciones 2, 3, 4 y 14, ubicadas en la parte interna y norte del estuario de Jambelí, lo que podría sugerir la influencia de los sistemas hídricos de la zona. Por otro lado, la estación 17 ubicada en la zona media del estuario interior del golfo de Guayaquil, se diferenció de las otras localidades, por presentar concentraciones más elevadas de nutrientes, lo que podría explicar el que no formó parte de ningún grupo identificado en el análisis de conglomerados. Esta estación estuvo ubicada frente al cantón Balao, donde se realizan actividades de agricultura y acuicultura. El último grupo estuvo integrado por las estaciones 5 y 6 , ubicadas en la parte externa del Golfo de Guayaquil, las cuales pueden haber sido influenciadas por la confluencia de las masas de agua transportadas por las diferentes corrientes marinas del área (Fig. 1A).

La abundancia promedio del fitoplancton registró variaciones entre $7.07 \pm 6.37$ y 1.95 $\pm 0.43 \times 10^{6}$ cel. $\mathrm{L}^{-1}$, correspondientes a las 


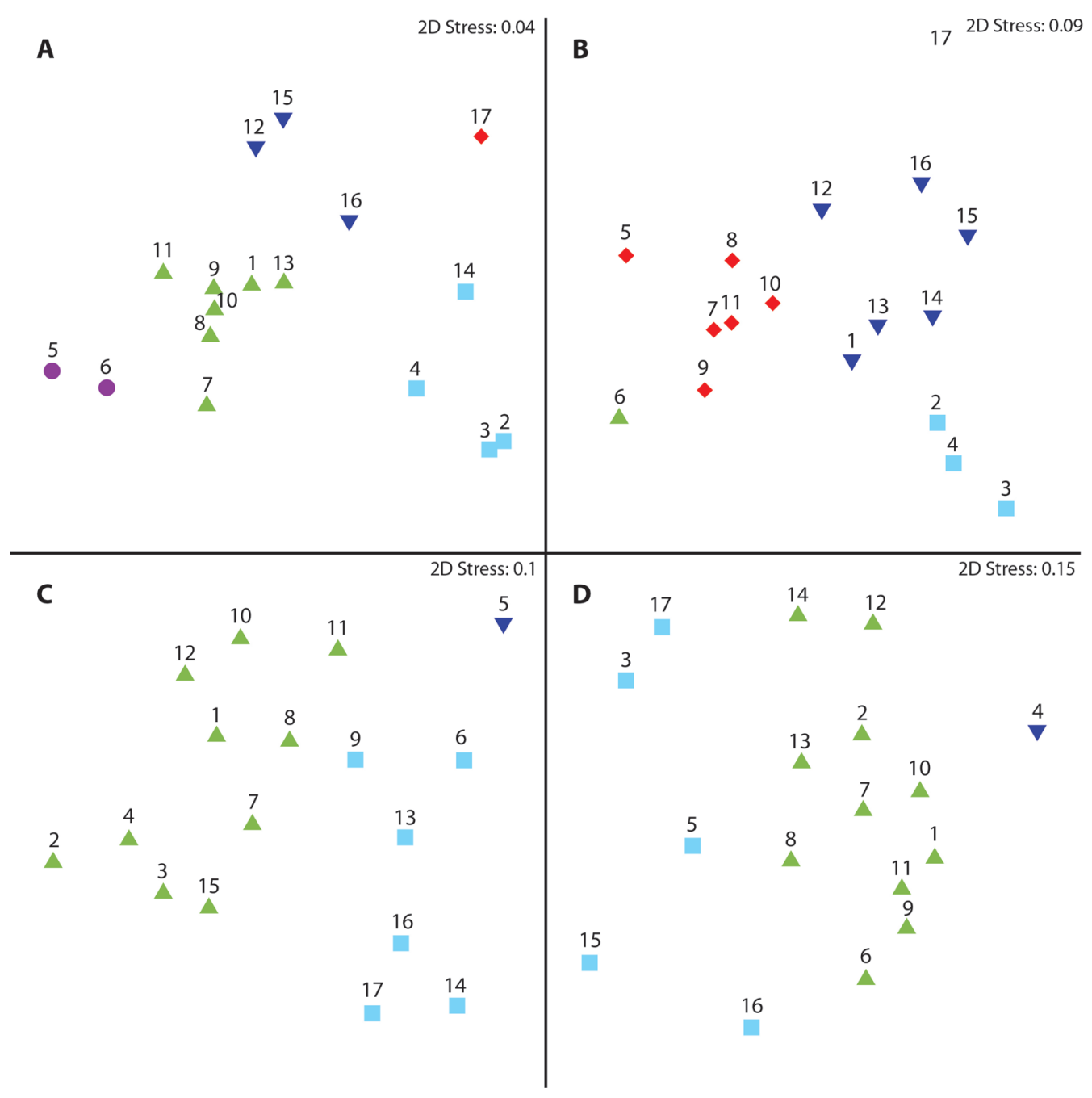

Fig. 1. Ordenamiento multidimensional a escala espacial de las variables bióticas y abióticas en la primera milla frente a la provincia de El Oro, Ecuador (junio-diciembre 2012) A) Variables ambientales, B) abundancias fitoplanctónicas, C) abundancias zooplanctónicas y D) abundancias ictioplanctónicas.

Fig. 1. Multidimensional scaling, spatial-scale of biotic and abiotic variables in the first mile in in front of El Oro province, Ecuador (June-December 2012) A) Environmental variables, B) phytoplankton abundances, C) zooplankton abundances y D) ichthyoplankton abundances.

estaciones 9 y 3 , ubicadas en el canal y estuario de Jambelí, respectivamente (APÉNDICE 1C). El componente fitoplanctónico estuvo dominado por bacilariofitas, que incluyeron especies estuarinas, costeras y marinas, como Lioloma pacificum, Eucampia cornuta, E. zoodiacus, Pseudo-nitzschia delicatissima, Skeletonema costatum, Chaetoceros decipiens, Paralia sulcata y Chaetoceros curvisetus, entre otras (APÉNDICE 2 A).

En la zona de estudio se definieron cinco grupos con base en el perfil de similaridad, el primero conformado por las estaciones 2 a 4, que correspondieron a la parte interna del estuario de Jambelí; el segundo y tercero, por las estaciones 6 y 17, respectivamente; el 
cuarto, conformado por las estaciones 1, 12 a 16 , y el último grupo, que incluyó a las estaciones ubicadas en el Canal de Jambelí, es decir 5, 7, 8 a 11 (Fig. 1B). Las principales especies responsables de estas agrupaciones fueron: $C h$. curvisetus, S. costatum, Ch. decipiens, Nitzschia longissima, P. delicatissima, Nitzschia sp., y $P$. sulcata.

La concentración zooplanctónica promedio fluctuó entre $4.44 \pm 7.01$ y $0.32 \pm 0.21 \mathrm{x}$ $10^{4}$ org. $\mathrm{m}^{-3}$, registradas en las estaciones 2 y 14 ubicadas en el canal de Jambelí y parte interna del estuario (APÉNDICE 1C), debido al aporte de varios taxones como son: Copepoda (Calanoida, Harpaticoida, Cyclopoida y Poecilostomatoida), Cirripedia, Copepodito, Decapoda (Brachyura), Cladocera, Aphragmophora y Myodocopida. Las estaciones se agruparon definiendo tres grupos, uno conformado por las estaciones $6,9,13,14,16$ y 17, otro integrado únicamente por la estación 5 , y el tercero compuesto por 1 a 4, 7, 8, 10 a 12 y 15 (Fig. 1C). Los principales taxones responsables de la conformación de los grupos fueron Cirripedia y Copepoda (Calanoida y Poecilostomatoida) (APÉNDICE 2B).

La abundancia promedio de larvas de peces registró al igual que el zooplancton su mínima concentración en la estación 3 con $160 \pm 110$ larvas. $10 \mathrm{~m}^{2}$ y su máximo valor en la estación 4 ubicada en el centro del canal de Jambelí, con $6610 \pm 10500$ larvas. $10 \mathrm{~m}^{2}$ (APÉNDICE 1C), las familias más representativas fueron Engraulidae, Scianidae y Haemulaidae. El perfil de similitud de larvas de peces entre estaciones, determinó la formación de tres grupos, el primero conformado por la estación 4 , ubicada en la parte interna del estuario de Jambelí; el segundo integrado por las estaciones 3, 5, 15 a 17 , y el resto de las estaciones, conformaron el tercer grupo (Fig. 1D). Las principales especies responsables por la formación de los grupos pertenecen a las familias Scianidae, Engraulidae, Haemulidae y Gobeiidae, específicamente a las especies Anchovia macrolepidota, Anchoa spp., y Achirus mazatlanus (APÉNDICE 2C).

De manera general, todas las variables abióticas a excepción de la salinidad mostraron diferencias espaciales significativas $(\mathrm{P}<0.05)$, mientras que en las abundancias relativas del fitoplancton, zooplancton y larvas de peces, no se observaron diferencias significativas $(\mathrm{P}>0.05)$.

Variación temporal: El máximo y mínimo de nitrito y nitrato se registró en septiembre y diciembre, con valores entre $0.083 \pm 0.097$ y $0.033 \pm 0.049 \mathrm{mg} . \mathrm{L}^{-1}$ en relación al primer nutriente y entre $0.212 \pm 0.193$ y $0.409 \pm 0.306$ mg. $\mathrm{L}^{-1}$ al segundo mencionado. En relación a silicato el máximo y mínimo del promedio fue determinado en junio y noviembre con fluctuaciones entre $5.441 \pm 2.986$ y $0.826 \pm 0.548$ $\mathrm{mg} . \mathrm{L}^{-1}$, respectivamente; mientras que el máximo de fosfato fue de $0.160 \pm 0.032$ durante junio y $0.097 \pm 0.040 \mathrm{mg} . \mathrm{L}^{-1}$ en julio (APÉNDICE 1D). El oxígeno disuelto promedio varió entre $5.46 \pm 1.93$ a $7.78 \pm 1.39$ mg.L $L^{-1}$ en junio y julio. La temperatura osciló entre $26.44 \pm$ 1.39 en junio a $24.06 \pm 0.79{ }^{\circ} \mathrm{C}$ en agosto; mientras que la salinidad registró sus máximos valores en octubre con $33.44 \pm 0.87$ ups y la menor concentración en junio con $30.23 \pm 2.91$ ups. Finalmente, la profundidad de la zona eufótica osciló entre $2.98 \pm 3.64$ a $7.82 \pm 8.73$ $\mathrm{m}$ en junio y diciembre (APÉNDICE 1E). En función del análisis de perfil de similitudes a escala temporal, junio se mostró diferente en relación a los otros meses, que conforman un solo grupo (Fig. 2A).

El fitoplancton registró abundancias promedios entre $1.66 \pm 0.96$ durante diciembre a $6.02 \pm 4.13 \times 10^{6}$ cel. $L^{-1}$ en octubre (APÉNDICE $1 \mathrm{~F})$. La determinación de la variación temporal del fitoplancton con base en la composición de especies, definió cinco grupos: junio-julio, agosto-septiembre, mientras que octubre, noviembre y diciembre se diferenciaron de los otros y entre sí (Fig. 2B). Las principales especies responsables de la formación de los grupos fueron $P$. delicatissima, S. costatum, C. decipiens, Ch. curvisetus y Coscinodiscus radiatus.

La abundancia zooplanctónica registró valores entre $0.12 \pm 0.10$ y $3.33 \pm 4.01 \times 10^{4}$ org. $\mathrm{m}^{-3}$, correspondiente a septiembre y junio, 


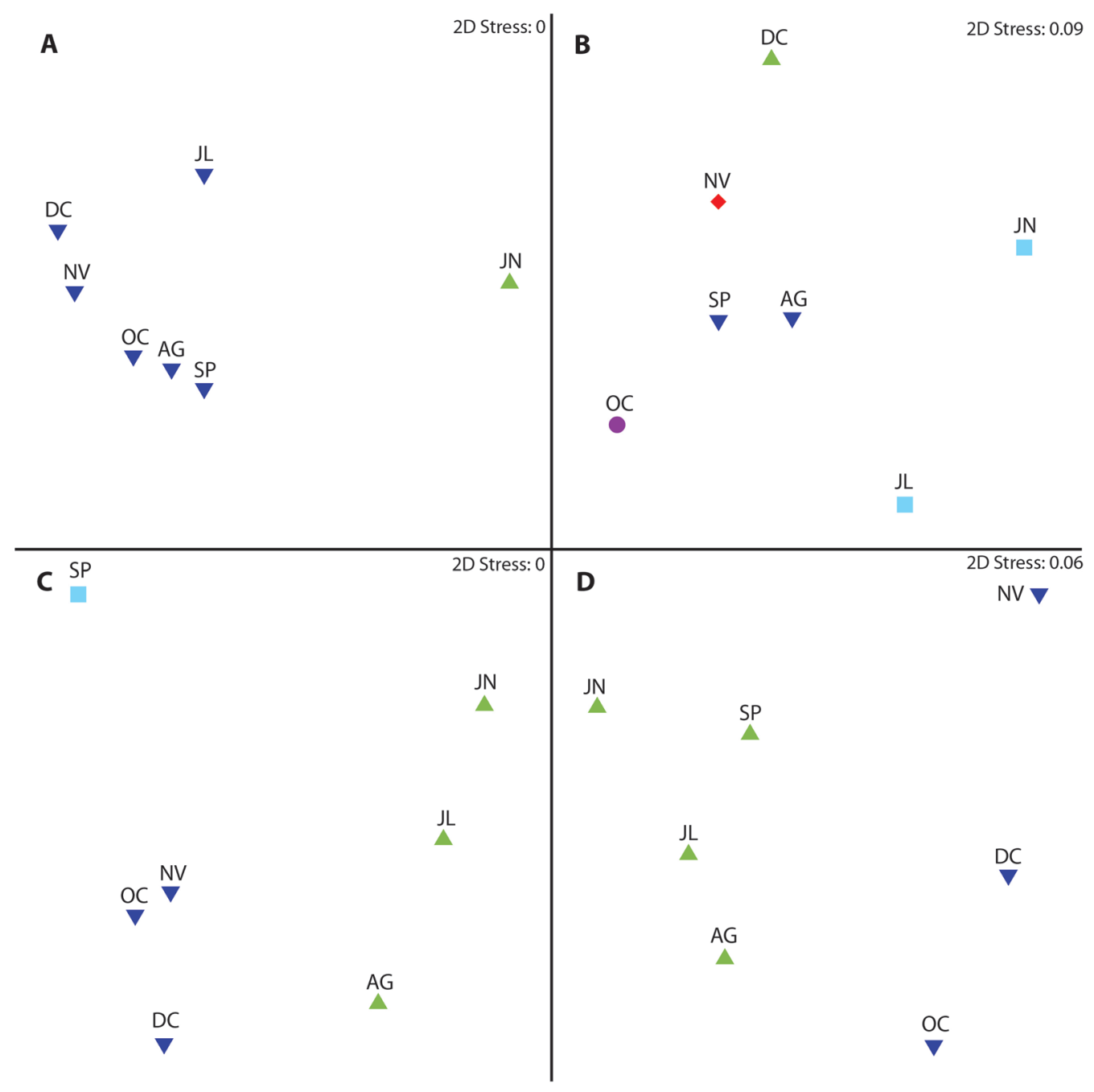

Fig. 2. Ordenamiento multidimensional a escala temporal de las variables bióticas y abióticas en la primera milla frente a la provincia de El Oro, Ecuador (junio-diciembre 2012) A) Variables abióticas, B) abundancias fitoplanctónicas, C) abundancias zooplanctónicas y D) abundancias ictioplanctónicas.

Fig. 2. Multidimensional scaling, spatial-scale of biotic and abiotic variables in the first mile in in front of El Oro province, Ecuador (June-December 2012) A) abiotic variables, B) phytoplankton abundances, C) zooplankton abundances y D) ichthyoplankton abundances.

respectivamente (APÉNDICE 1F). El análisis de perfil de similitud definió tres grupos: junio a agosto, octubre a diciembre y septiembre, este último como grupo independiente (Fig. 2C). Los principales taxones responsables de la tendencia observada fueron Copepoda (Calanoida y Harpaticoida), Cirripedia, Cladocera y Brachyura.
Las concentraciones de larvas de peces tuvieron su mayor densidad promedio en diciembre con $5420 \pm 6180$ larvas. $10 \mathrm{~m}^{2}$, mientras que en agosto se registró el menor valor con $630 \pm 980$ larvas. $10 \mathrm{~m}^{2}$ (APÉNDICE 1F). La similitud temporal de estos organismos determinó la formación de dos grupos, junio a septiembre conformaron el primer grupo y 
los meses restantes el segundo (Fig. 2D). Las principales especies de larvas de peces responsables de la similitud entre meses fueron Anchovia spp., y A. macrolepiota, pertenecientes a la familia Engraulidae.

Se determinaron diferencias temporales significativas $(\mathrm{P}<0.05)$, entre las variables abióticas con excepción de la profundidad de la zona eufótica, mientras que de las bióticas únicamente las abundancias relativas de larvas de peces, mostraron diferencias significativas $(\mathrm{P}<0.05)$.

\section{DISCUSIÓN}

Si se considera que la productividad, ciclo de nutrientes y el acoplamientos de los procesos ecológicos en aguas costeras son influenciadas por la geomorfología y características geofísicas de las mismas, y que la calidad del agua es afectada por las actividades que se dan al interior de las cuencas hidrográficas continentales (Twelly et al., 1999 citado en Montaño, 2010; Twilley y Day, 1999), la primera milla náutica frente a la Provincia de El Oro, corresponde a lo esperado para un ecosistema estuarino con alta productividad, coincidiendo con lo reportado por Bonilla-Coello, Pluás, \& Campuzano (2002).

La influencia de los factores antes mencionados, además de las actividades antrópicas desarrolladas, principalmente agricultura y acuicultura (Montaño, 2010), se evidencian en los patrones de distribución espacial y temporal observados, que determinan la formación de tres áreas que estarían asociadas a corrientes oceánicas, costeras y de marea, así como también a patrones de circulación determinados por las características hidrológicas y morfológicas del área (isla Puná, Archipiélago de Jambelí, Estero Salado, canales de Cascajal y Jambelí y río Guayas), estas zonas son:

El interior del Archipiélago de Jambelí y frente a la isla Puná, influenciada por las actividades productivas desarrolladas en sus márgenes (acuicultura) y la hidrografía del área.

Otra zona, conformada por las estaciones ubicadas en el estuario exterior que incluye inmediaciones de la isla Santa Clara, donde la influencia oceánica es evidente.

Finalmente, el canal de Jambelí, caracterizado por procesos de mezcla, producto del flujo de mareas.

En relación al fitoplancton, se presentó un ensamblaje compuesto por especies oceánicas, neríticas y estuarinas, que en función de su abundancia y condiciones ambientales, se alternan en dominancia en las diferentes áreas; lo que coincide con lo reportado por otros autores en éste y otros sistemas estuarinos (Karthik, Arun Kumar, Sai Elangovan, Siva Sankar, \& Padmavati, 2012; Brogueira, Oliveira, \& Cabeçadas, 2007; Torres, Calderón, Mero, \& Franco, 2004; Cajas, Coello, \& Domínguez, 1998). Sin embargo, el zooplancton podría influenciar la densidad y distribución del fitoplancton a través de interacciones tróficas como pastoreo, y en menor medida por medio de la remineralización de sus heces (Xing, 2013; Møller, 2005).

Los copépodos mostraron una distribución uniforme; sin embargo, la mayor concentración de larvas de cirripedios, decápodos y braquiuros en las zonas estuarinas, sugiere una tendencia a la zonación de estos taxones. El ictioplancton registrado durante el estudio, corresponde principalmente a especies con hábitos alimenticios basados en copépodos e incluso algunas microalgas, entre las que se encontraron diatomeas como los géneros Skeletonema, Nitzschia, Chaetoceros, Rhizosolenia e incluso dinoflagelados y cianobacterias (Lima \& Barletta, 2016).

En cuanto a la distribución del ictioplancton, los engráulidos, representados principalmente por Anchoa spp., y Anchovia macrolepidota, dominaron en toda el área de estudio. Distribución que estaría explicada por ser esta una familia representativa de zonas estuarinas, al igual que la familia Gobiidae; mientras que los ciánidos corresponden a especies demersales con desoves en áreas costeras, estuarios y bahías (Ocaña-Luna \& SánchezRamírez, 2003; Fahay, 1983).

Estos resultados coinciden con lo establecido por Peribonio, Repelin, Luzuriaga de 
Cruz, Hinostroza y Villaroel (1981), quienes definieron la existencia de dos sectores de producción biológica elevada durante la época seca en el golfo de Guayaquil, uno de los cuales se ubica al sur de la Isla Puná, en la zona de ascenso de las aguas de origen marino, y su encuentro con las aguas del río Guayas, así como también una circulación de tipo estuarino que permite al Golfo recibir simultáneamente los elementos fertilizantes del continente y del océano.

Temporalmente, mayo registró precipitaciones que excedieron los valores normales (BAC 260, 261, 2016), lo que habría determinado altas concentraciones de nutrientes, disminución de salinidad y de la zona fótica, lo que explicaría la diferencia estadística encontrada entre junio y el resto del período de muestreo. A partir de julio, se observó una disminución progresiva de nutrientes hasta diciembre, resultado del consumo del fitoplancton. Sin embargo, la disponibilidad de nutrientes existente permitió a lo largo de todo el período de estudio mantener su composición y abundancia.

En relación con la composición fitoplanctónica, se registró alternabilidad entre las especies más representativas, pero sus abundancias permiten establecer de julio a septiembre la dominancia de alguna de ellas. Sin embargo, de octubre a diciembre todas las especies incrementaron sus densidades, sin que se establezcan dominancias específicas, lo que determinó las diferencias temporales observadas.

La composición del zooplancton fue similar durante todo el periodo, pero su abundancia determinó dos periodos, uno de junio a agosto $\mathrm{y}$ otro de octubre a diciembre, con mayor y menor concentración, respectivamente; mientras que en septiembre las abundancias decaen considerablemente, lo que podría ser explicado por patrones poblacionales específicos de las diferentes especies registradas, sin descartar la influencia de corrientes de marea y de los flujos de los ríos, que pueden enmascarar la estacionalidad del zooplancton (Marques, 2005). Estos resultados deben ser interpretados con precaución debido a que las poblaciones estuarinas son extremadamente dependientes del efecto de las mareas (Marques, 2005), y que el análisis fue realizado a nivel de taxones gruesos por lo que puede haber estacionalidad a nivel de especie que no ha sido detectada.

El ictioplancton también mostró dos periodos, de junio a septiembre, y octubre a diciembre, condición que se origina con el incremento progresivo de larvas de $A$. macrolepidota. Esta especie forma grandes cardúmenes en las aguas estuarinas de la desembocadura de ríos donde se alimenta de fitoplancton, zooplancton, y de huevos y larvas de otras especies pelágicas (Galván-Magaña, Gutiérrez-Sánchez, AbitiaCardenas, \& Rodríguez-Romero, 2000).

\section{AGRADECIMIENTOS}

Al Instituto Nacional de Pesca (INP) y a la Secretaria Nacional de Educación Superior, Ciencia, Tecnología e Innovación (SENESCYT), por el desarrollo y financiamiento brindado a la ejecución del Proyecto "Condiciones Biológicas-Pesqueras y Artes de Pesca en la franja Marino Costera dentro la Primera Milla Náutica de la Costa Ecuatoriana“, así como también a todos y cada uno de los profesionales del INP que participaron en la colecta, análisis e ingreso de datos correspondientes a las variables, físicas, químicas y planctónicas.

\section{RESUMEN}

El Oro incluye diferentes sistemas costeros y estuarinos y es parte del Golfo de Guayaquil. Estas áreas proporcionan diferentes servicios ecosistémicos, pero con el aumento de la población y de las diversas actividades económicas, los recursos naturales locales poco estudiados pueden verse afectados negativamente. Es por ello que esta investigación tuvo como objetivo describir la dinámica del plancton, productividad y ecológica de esta área durante la estación seca de 2012, para lo cual se establecieron 17 estaciones para estudiar la distribución espacial y variación mensual de los nutrientes inorgánicos, temperatura, oxígeno disuelto, salinidad, zona fótica, abundancia y composición del plancton y larvas de peces, con métodos estándar. Se establecieron patrones espaciales y temporales mediante análisis de conglomerados y de ordenamiento multidimensional. Los resultados obtenidos de las variables bióticas y abióticas analizadas determinaron un patrón característico de un ecosistema estuarino productivo, así como también la existencia de áreas específicas asociadas a condiciones 
naturales y antrópicas, que son: a) interior del Archipiélago de Jambelí y frente a la isla Puná, influenciada por las actividades productivas y la hidrografía del área; b) una zona, conformada por las estaciones ubicadas en el estuario exterior donde la influencia oceánica es evidente y c) el canal de Jambelí, caracterizado por procesos de mezcla producto del flujo de mareas. Lo que se evidenció en la presencia de fitoplancton oceánico, nerítico y estuarino; copépodos con distribución uniforme en el área de estudio y dominancia de la familia Engraulidae representativa en este tipo de ecosistema acuático en lo referente a larvas de peces. Temporalmente, mayo registró precipitaciones que excedieron los valores normales, lo que habría determinado las altas concentraciones de nutrientes, la disminución de salinidad y de la zona fótica, registradas en este mes. Sin embargo la disponibilidad de nutrientes existente permitió a lo largo de todo el período de estudio mantener la composición y abundancia del fitoplancton con alternancia de especies representativas y que el zooplancton registre periodos de mayor (junio a agosto) y menor (octubre a diciembre) abundancia, lo que sería resultado de patrones poblacionales específicos de las especies presentes e influencia de la marea y flujos del río aportante; comportamiento similar registró el ictioplancton resultado de las concentraciones de Anchoa macrolepidota, especie característica de este ecosistema. Estos patrones de distribución espacio-temporal, permiten establecer el desarrollo de múltiples interacciones abióticas y bióticas que determinan diferentes estrategias para mantener la productividad del área, por lo que se recomienda desarrollar esta investigación durante la estación lluviosa en el Ecuador.

Palabras clave: plancton; Golfo de Guayaquil; estacionalidad seca; variables bióticas y abióticas.

\section{REFERENCIAS}

Aguirre, W. E., \& Shervette, V. R. (2005). Morphological diversity of the Cynoscion group (Perciformes: Sciaenidae) in the Gulf of Guayaquil region, Ecuador: a comparative approach. Environmental Biology of Fishes, 73(4), 403-413.

Ahlstrom, E. H. (1972). Kinds and abundance of fish larvae in the Easter Tropical Pacific on the second multivessel EASTROPAC survey, and observations on the annual cycle of larval abundance. Fisheries Bulletin. U. S., 70(4), 1153-1242.

Al-Kandari, Al-Yamani, F., \& Al-Rifaie, K. (2009). Marine Phytoplankton Atlas of Kuwait's waters. Kuwait: Institute for Scientific Research.

Beltrán-León, B. \& Ríos, R. (2000). Estadios tempranos de peces del Pacífico Colombiano Tomo 2. Instituto Nacional de Pesca y Acuacultura. Buenaventura-Colombia.
Björnberg, T. A. (1981). Copepoda. En D. Boltovskoy (Ed), Atlas del zooplancton del Atlántico Sudoccidental y métodos de trabajo con el zooplancton marino (pp. 587-679). Mar del Plata. Publicación especial del Instituto Nacional de Investigaciones y Desarrollo Pesquero (INIDEP).

Boletín de Alerta Climática (BAC) 260. (2016). Instituto Oceanográfico de la Armada - BAC - El Niño. Inocar. mil.ec. Recuperado de: https://www.inocar.mil.ec/ web/index.php/boletines/bac

Boletín de Alerta Climática (BAC) 261. (2016). Instituto Oceanográfico de la Armada - BAC - El Niño. Inocar. mil.ec. Recuperado de: https://www.inocar.mil.ec/ web/index.php/boletines/bac

Boltovskoy, D. (1981). Atlas del Zooplancton del Atlántico Sudoccidental y métodos de trabajo para el zooplancton marino (1 st ed.). Mar del Plata: Publicación Especial Instituto Nacional de Investigación Pesquera.

Bonilla-Coello, M., Pluás, F., \& Campuzano, J. (2002). Condiciones de plancton en una estación fija: Puerto el Morro-Playas, Golfo de Guayaquil, 200-2002. Acta Oceanográfica del Pacífico, 11(1), 3-12.

Brogueira, M., Oliveira, M., \& Cabeçadas, G. (2007). Phytoplankton community structure defined by key environmental variables in Tagus estuary, Portugal. Marine Environmental Research, 64(5), 616-628. DOI: 10.1016/j.marenvres.2007.06.007

Cajas, L., Coello, D., \& Domínguez, C. (1998). Comunidades del fitoplancton y zooplancton en el Estuario Interior del Golfo de Guayaquil. In Comportamiento temporal y espacial de las características físicas, químicas y biológicas del Golfo de Guayaquil y sus afluentes Daule y Babahoyo entre 1994-1996 (1st ed., pp. 261-284). Guayaquil: Instituto Nacional de Pesca.

Calderón, G. (2011). Catálogo de huevos y larvas de peces colectadas en aguas ecuatorianas. Boletín Especial, 2(4), 118.

Carpenter, J. H. (1965). The Accuracy of the Winkler Method for Dissolved Oxygen Analysis1. Limnology and Oceanography, 10(1), 135-140. DOI: 10.4319/ 1o.1965.10.1.0135

Clarke, K. R., \& Gorley, R. N. (2005). PRIMER v.6: User Manual / Tutorial. PRIMER-E Ltda., Plymouth, UK.

Coello, S., \& Altamirano, M. (2007). Buenas prácticas de Aprovechamiento y Uso de los Recursos Costeros en Ecuador. Una guía para su sistematización y elementos a considerar para impulsarlas (1st ed.). Quito: Avina-Ecobiotec-Ecocostas-Ministerio Del Ambiente-Conservación Internacional.

Cucalón, E. (1996). Primera Parte: Oceanografía y Sistemas Físicos. In Sistemas Biofisicos del Golfo de Guayaquil (1st ed., pp. 1-109). Quito: Comisión 
Asesora Ambiental de la Presidencia de la República del Ecuador.

Dominguez, L., \& Fockedey, N. (2001). Variación diurna del hiperbentos en la playa arenosa de San Pedro de Manglaralto (Prov. Guayas, Ecuador). (Tesis de pregrado). Escuela Superior Politécnica del Litoral, Ecuador.

Fahay, M. (1983). Guide to the Early Stages of Marine Fishes Occurring in the Western North Atlantic Ocean, Cape Hatteras to the Southern Scotian Shelf. Journal of Northwest Atlantic Fishery Science, 4, 3-423. DOI: 10.2960/j.v4.a1

Galván-Magaña, F., Gutierrez-Sánchez, F., Abitia-Cardenas, L., \& Rodríguez-Romero, J. (2000). The distribution and affinities of the shore fishes of the Baja California Sur lagoons. In Aquatic Ecosystems of Mexico: Status and Scope. Ecovision World Monograph Series.

Hurtado, M. A., Hurtado-Domínguez, L. M., HurtadoDomínguez, L., Soto, L., \& Merizalde, M. A. (2010). Áreas costeras y marinas protegidas del Ecuador (1st ed.). Quito: Ministerio del Ambiente.

Jiménez, R. (1996). Segunda Parte: Biología, Ecología y Acuacultura. In Sistemas Biofisicos del Golfo de Guayaquil (1st ed., pp. 111-223). Quito: Comisión Asesora Ambiental de la Presidencia de la República del Ecuador.

Karthik, R., Arun Kumar, M., Sai Elangovan, S., Siva Sankar, R., \& Padmavati, G. (2012). Phytoplankton Abundance and Diversity in the Coastal Waters of Port Blair, South Andaman Island in Relation to Environmental Variables. Journal of Marine Biology \& Oceanography, 01(02). DOI: 10.4172/2324-8661.1000102

Kirk, J. (1994). Light and photosynthesis in aquatic ecosystems (1st ed.). Cambridge: Cambridge University Press.

Licea, S., Moreno, J. L., Santoyo, H., \& Figueroa, G. (1995), Dinoflagelados del Golfo de California. (1st ed.). México. Universidad Autónoma De Baja California Sur.

Lima, A., \& Barletta, M. (2016). Lunar influence on prey availability, diet shifts and niche overlap between Engraulidae larvae in tropical mangrove creeks. Journal of Fish Biology, 89(4), 2133-2152.

Marques, S. (2005). Zooplankton and ichthyoplankton communities in a temperate estuary: spatial and temporal patterns. Journal of Plankton, 28(3), 297-312. DOI: $10.1093 /$ plankt/fbi126

Møller, E. (2005). Sloppy feeding in marine copepods: prey-size-dependent production of dissolved organic carbon. Journal of Plankton Research, 27(1), 27-35. DOI: $10.1093 /$ plankt/fbh147
Montaño, M. (2010). Ecosistema Guayas (Ecuador): Recursos, Medio Ambiente Y Sostenibilidad En La Perspectiva del Conocimiento Tropical. (Tesis Doctorado) Universidad Miguel Hernández De Elche, Alicante, España.

Moreano, H. (1983). Interacción Océano - Atmósfera sobre la zona costera del Ecuador. Acta Oceanográfica del Pacífico, 2(1), 1-11.

Moreno, J. L., Licea, S., \& Santoyo, H. (1996). Diatomeas del Golfo de California. (1st ed.) México: Universidad Autónoma De Baja California Sur.

Ocaña-Luna, A., \& Sánchez-Ramirez, M. (2003). Diversity of ichthyoplankton in Tampamachoco Lagoon, Veracruz, México. Anales del Instituto de Biología Serie Zoología, 74(2), 179-193.

Palma, A. T., Henríquez, L. A., \& Ojeda, F. P. (2009). Phytoplanktonic primary production modulated by coastal geomorphology in a highly dynamic environment of central Chile. Revista de Biología Marina y Oceanografia, 44(2), 325-334.

Peribonio, R., Repelin, R., Luzuriaga de Cruz, M., Hinostroza, D., \& Villaroel, J. (1981). Estudio ecológico del Golfo de Guayaquil abundancia, ciclos nictamerales y relaciones entre el estuario del Río Guayas y el océano. Boletín Científico y Técnico Del Instituto Nacional De Pesca, IV(2), 30 p.

R Core Team (2014). R: A language and environment for statistical computing. R Foundation for Statistical Computing, Vienna, Austria. http://www.R-project. org

Reguera, B., Alonso, R., Moreira, A., \& Méndez, S. (2011). Guía para el diseño y puesta en marcha de un plan de seguimiento de microalgas productoras de toxinas (1st ed., p. 65). París Viena: Comisión Oceanográfica Intergubernamental. Recuperado de: http://unesdoc. unesco.org/images/0021/002145/214510s.pdf

Rice, E. W., Baird, R. B., Eaton, A. D., \& Clesceri, L. S. (2012). Standard methods for the examination of water and wastewater. American Public Health Association, American Water Works Association, and Water Environment Federation.

Rodríguez, A., Hernández, R., D., \& Lizárraga, I. G. (2008). Catálogo de Microalgas de las Lagunas Costeras de Sinaloa. México: Universidad Nacional Autónoma de México.

Siqueiros-Beltrones, D. A. (2002). Diatomeas bentónicas de la península de Baja California; diversidad y potencial ecológico. La Paz: Instituto Politécnico Nacional y Universidad Autónoma de Baja California del Sur.

Smith, E. \& Richardson, S. (1979). Técnicas Modelo para las Prospecciones de huevos y larvas de peces pelágicos (1st ed., No. 75). Roma: Organización 
de las Naciones Unidas para la Agricultura y la Alimentación.

Soler, B. A., Pérez, A. M., \& Aguilar, G. E. (2003). Diatomeas de las Costas del Pacífico de Panamá. Panamá: Universidad de Panamá.

Suárez-Morales, E. (1995). Clave ilustrada para la identificación de los copépodos pláncticos de la Bahía de Chetumal, México. Avances Científicos, 12, 16-24.

Suárez-Morales, E. (1996). Clave de identificación ilustrada de los copépodos pláncticos (Crustacea) de la Bahía de la Ascensión, Chetumal, Q. Roo. Avances Cientificos, 17, 25-39.

Tomas, C. (Ed.). (1996). Identifying marine diatoms and dinoflagellates. New York, USA: Academic Press.

Torres, G., Calderón, T., Mero, M., \& Franco, V. (2004). Procesos Planctónicos en el Golfo de Guayaquil
(Campo Amistad) Julio-Agosto 2001. Acta Oceanográfica del Pacífico, 12, 81-91.

Twilley, R. R., \& Day, J. W. Jr. (1999). The productivity and nutrient cycling of mangrove ecosystems. In A. Yáñez-Arancibia \& A. L. Lara-Domínguez (Eds.), Ecosistemas de Manglar en América Tropical (pp. 127-15). Instituto de Ecología A.C. México, UICN/ ORMA, Costa Rica, NOAA/NMFS Silver Spring MD USA.

Vera, L., Lucero, M., \& Mindiola, M. (2009). Caracterización Oceanográfica de la costa central ecuatoriana entre la Punta del Morro y Jaramijó, Ecuador. Boletín Oceanográfico de Pacífico, 9(11), 7-17.

Xing, Ch. (2013). Modelling the life cycle dynamics of Acartia clausi: a key copepod species in the North Sea. (Diss). Universität Hamburg Hamburg.

Zar, J. (2009). Biostatistical analysis (1st ed.). Prentice-Hall. 